\title{
Experimental Characterization of Drag Coefficient of an UAV Recovery Parachute
}

\author{
Vu Dinh Quy, Le Thi Tuyet Nhung*, Tran Minh Duy Dat \\ Hanoi University of Science and Technology, Hanoi, Vietnam \\ *Email: nhung.lethituyet@hust.edu.vn
}

\begin{abstract}
Parachute recovery systems are proved to be an efficient method to recovery and rescue unmanned aerial vehicles (UAV) as it follows most requirements of reliability and airworthiness in flights. Parachutes are key components of the recovery systems and the drag coefficient of parachutes plays a crucial role in evaluating parachute's performance. The purpose of the research is to determine and compare the impact of some factors on aerodynamic drag force during the inflation of a parachute. The canopy's shape (flat circular type and extended skirt 10\% flat type), of the length of suspension lines (be in proportion to nominal diameter from 0.6 to 1.5) are considered. Measurement of the drag force of the parachute models is carried out in an open return wind tunnel. Experimental results show that flat circular canopy has a higher drag coefficient than extended skirt $10 \%$ flat model in the range of low speed from 3 to $6 \mathrm{~m} / \mathrm{s}$. However, when wind speed is greater than 6 $\mathrm{m} / \mathrm{s}$, the drag coefficients of both two parachute types are nearly 0.85. In terms of the suspension line, the longer length would significantly raise the coefficient of drag force.
\end{abstract}

Keywords: UAV, parachute recovery system, parachute, drag coefficient.

\section{Introduction}

An unmanned aerial vehicle (UAV) known as a drone, is a pilotless aircraft, which is flown without pilot-in-command on-board and is either remotely and fully controlled from another place (ground, another aircraft, space) or programmed and fully autonomous. UAVs initially are developed for military reconnaissance, however, they are expanding their applications in other fields such as communities, law enforcement, scientific projects, agriculture and so on [1]. An unmanned aircraft system commonly include a number of cutting-edge and expensive electronic parts and components like sensors, central controllers, batteries, actuators, optical equipment, etc [2]. In addition, the collected data from the flight of drones is an important object which is often needed to prevent it from any unexpected access and damage. Safe and airworthy requirements for components as well as UAVs as a whole need to take steps to maintain stability and advantages.

Civil drones have a dramatic increase in the whole world, which leads to a challenge for authorities to control drones' operations. Therefore, these could have potential risks for civilians if drones are used in residential or urban areas. In the future, governments can impose new regulations to guarantee that the flight of drones would have absolutely no harm [3].

Consequently, recovery systems are gradually recognized as the most indispensable component for a
UAV to solve most problems mentioned. Recover systems are classified based on a variety of constructive solutions: manual and automatic command, standard or emergency procedure, shock or without shock (mechanical, pyrotechnical/pneumatic); maneuverable or non-maneuverable, mounted on aerial systems (parachute, airbag) or ground systems (net, skyhook) [2, 4].

The parachute recovery system is one of the most popular recovery systems used for drones because its benefits in operation has been proven. This system can be designed to become a modulus equipment, which allows it to be installed directly in many kinds of drones with a large range of take-off weight. The rescue and recovery process using parachutes can be deployed rapidly on aerial vectors so it occurs at unready positions. Methods using recovery nets or skyhooks need other mechanical components and a support group so it takes crews a lot of time to set up and deploy. Besides, the parachute recovery system can slow down the speed of UAVs to the allowable value while net or skyhook systems catch the drones at high speed which leads to mechanical damages of wings or propulsions. Therefore, parachutes are also safer and smoother than others. However, strong wind is an environmental condition having effects on the parachute's inflation and descent and hence it is more difficulty for crews to recover drones when appearing in complex situations $[2,5]$.

ISSN 2734-9373

https://doi.org/10.51316/jst.152.ssad.2021.31.2.9

Received: January 5, 2021; accepted: August 25, 2021 


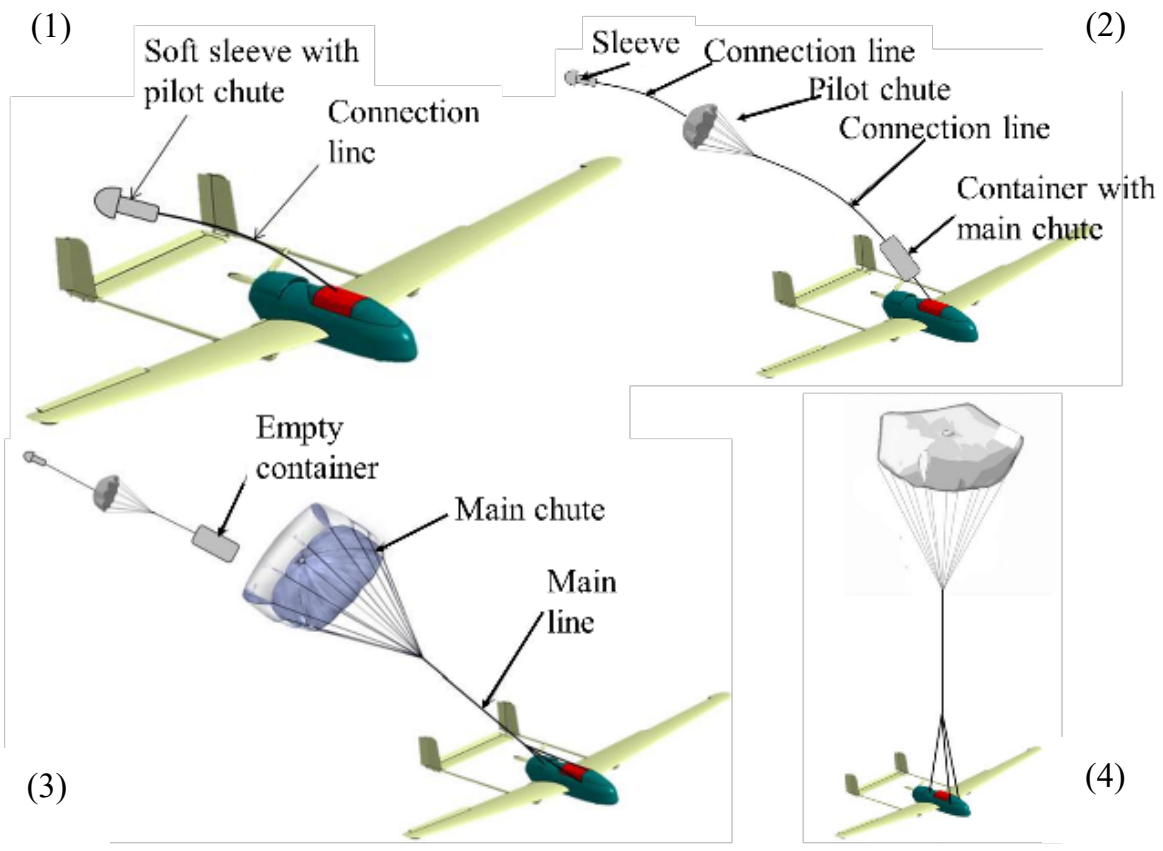

Fig. 1. The deployment process of a parachute recovery system [5]: (1) Launching a pilot chute; (2) Deploying the main chute container; (3) Opening the main chute; (4) Steady descent of UAV.

Primary components of the parachute recovery system can contain a parachute, a container for package and launching and a control system including a receiver, a transmitter and a central processor. The whole process from starting the deployment of the parachute to the full inflation (shown in Fig. 1) is divided into four steps following: 1. Opening container and launching the pilot chute; 2 . Deploying the main chute; 3 . The main chute is fully inflated; 4 . The drone is in steady descent [5].

The drag coefficient which is dimensionless and nonlinear is a typical range of values for a specific parachute. In steady descent, the velocity of parachutes is constant which means that the dynamic pressure $q=1 / 2 p v^{2}$ is a fixed value. Hence, it can be clearly seen that drag force relies on the drag area $C_{D_{0}} S_{o}$. For a given drag area, the higher value of the drag coefficient $C_{D_{0}}$ is, the smaller the nominal area $S_{o}$ can be. Thus, the drag coefficient indicates how effectively a parachute canopy produces drag with a minimum of cloth area thereby minimizing weight and volume. The drag coefficient is dependent on many nonlinear characteristics so designers need to consider carefully to give a drawing with a good balance of factors [6].

In many decades, there is a large amount of research investigating all characteristics of parachutes and the flow field around them by simulation and experiment manners. Air Materiel Command performed a series of drop and 12-feet vertical wind tunnel tests to research the effect resulting from many factors on the parachutes' performance. Those factors consisting of design, shape, porosity and etc. were evaluated by experiments of various types of parachutes with the canopy area of $1600 \mathrm{in}^{2}$ and the same suspension line length [7]. Similarly, a program where various types of parachute models experienced drop tests was conducted at Goodyear Airdock in Ohio, USA. Models which have a total canopy area of $16,000 \mathrm{in}^{2}\left(10.32 \mathrm{~m}^{2}\right)$ were tested to compare their curve of the drag coefficient versus the vertical descent velocity. The report illustrated the impact caused by the length of suspension lines on the drag coefficient and gliding and oscillating tendency in the motion of parachutes [8]. Johari and Levshin utilized a water tunnel to study the interaction of vortex around solid round parachute canopies. Canopy models with three different diameters attached to a forebody were tested to observe the vortex in the inflation process of parachutes and measure the time-averaged drag force. [9] Jin et al. used the Stereoscopic Particle Image Velocimetry (Stereo-PIV) technique to investigate the flow field around many different parachute canopies. The results illustrated that the geometry of parachutes had a considerable impact on the flow structure of the turbulent wake. Meanwhile, the Reynold number was pointed out that it did not affect the profile of velocity, vorticity and Reynolds number [10-12].

Moreover, numerical solutions have rapidly developed and been applied to research and verify aerodynamic and design characteristics of parachutes. Stein et al. applied a parallel computational method to simulate 3-D parachute fluid-structure interaction for a 
round parachute (T-10 personal parachute). In detail, a deforming-spatial-domain/ stabilized space-time (DSD/ SST) finite element formulation was used for fluid dynamics, while structure dynamics was solved by a finite element formation obtained from the principle of virtual work. Because of the incompatibility of FD and SD meshes, the coupling problem was dealt with by an advanced algorithm [13]. $\mathrm{Yu} \mathrm{Li}$ et al. developed the simplified arbitrary Lagrangian-Eulerian fluid-structure interaction (SALE/FSI) method to simulate the inflation of a parachute which was built as a star-shaped folded model. The research calculated and showed the number of numerical results such as opening load, drag performance, swinging angle, etc. which are in accordance with those from wind tunnel tests. Moreover, the method predicted the time-depended change in canopy shape, motion, structure and flow field around the parachute [11]. The arbitrary Lagrangian-Eulerian (ALE) approach continues to be used and combined with interface tracking methods to simulate the supersonic parachute inflation in the work of Xue Yang et al. Results obtained from numerical models such as maximum Root Mean Square (RMS) drag, general canopy shape and the smallest canopy projected areas in the terminal descent state were consistent with experiments carried out in the wind tunnel [14].

In this paper, measurements of the drag force of parachute models with the two different types of canopies and the change in the suspension line length will be carried out in a wind tunnel. The results are used to calculate the drag coefficient and characterize the effects of some design factors on the drag coefficient.

\section{Theory}

\subsection{Calculating the Size of Parachutes}

Let consider a parachute system in descent, that is applied by the total drag of the parachute, the load, $D_{T}$, and the weight of the load and the parachute assembly, $W_{T}$ [6].

$$
\overrightarrow{W_{T}}+\overrightarrow{D_{T}}=m \cdot \vec{a}
$$

The total drag force is contributed by both payload and parachute. The drag of load $D_{l}$ can be neglected in relation to the large drag of parachute $D_{p}$ which could be calculated by the equation:

$$
D_{p}=\frac{1}{2} \rho v^{2} C_{D_{o}} \cdot S_{o}
$$

where $\rho$ is air density; $v, C_{D_{o}}$ and $S_{o}$ are the velocity, nominal drag coefficient and area of parachutes, respectively [6].

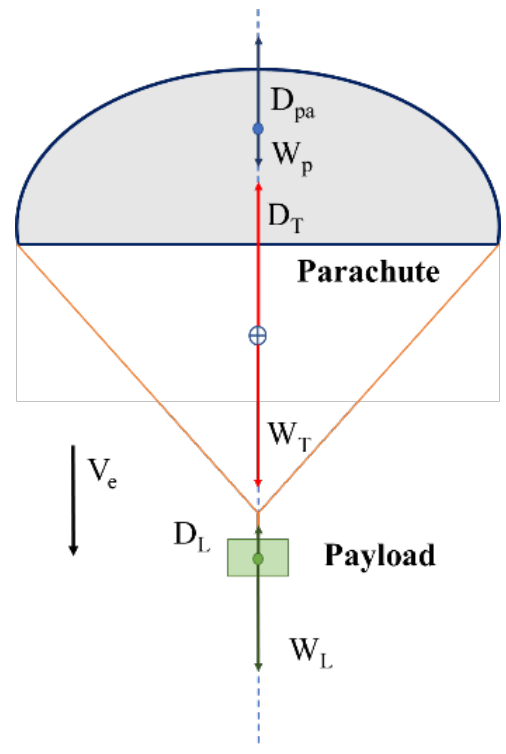

Fig. 2. Forces acting on a parachute in the steady descent

In steady descent, the unaccelerated parachute is an equilibrium of the forces, which means that the total weight is equal to the drag of parachutes. Thus, the canopy surface area $S_{0}$ can be calculated [6]:

$$
S_{0}=\frac{2 W_{T}}{\rho C_{D o} v^{2}}
$$

In case, we consider the area $S_{0}=\pi D_{o}^{2} / 4$. Thus, the equation to determine the nominal diameter is:

$$
D_{o}=\sqrt{\frac{8 W_{T}}{\pi \rho C_{D o} v^{2}}}
$$

\subsection{Drag Coefficient}

The drag force is the most important characteristic to evaluate the parachute's performance. As mentioned in previous sections, a high drag coefficient can optimize the weight and the volume of parachutes. It is vital that an additional system is installed on the drones. Depending on the object using the parachute, the consideration to choose the type of canopy would be discussed. The circular, flat, solid textile parachutes give superior performance characteristics in drag at the descent with low speed. While slotted parachutes could be mainly used for the application in the supersonic rate of descent, others have gliding mode which is in accordance with amphibious tasks in both military and civil applications.

Previous tests indicate that a decrease of canopy porosity and an increase of suspension-line length $L_{e}$ are the prime reasons causing the growth of inflated canopy diameter and are associated with rising drag coefficient. Normally, if these lines increase their length, the canopy opens wider with the larger inflated 
area $S_{p}$ and projected diameter $D_{p}$. Drag coefficient can be added if the ratio of suspension-line length and nominal diameter $D_{o}$ is up to 2.0. Nevertheless, in practice, there is a given optimized length of suspension line for a specific parachute, which means that there is only a slight increase in drag force when extending the suspension lines [6].

\subsection{Experiments of Parachutes in the Wind Tunnel}

In practice, some experiments are conducted to figure out the aerodynamic characteristics by full-scale parachute models. The most popular manner is drop tests in which prototypes are deployed and inflated in the natural environment. Besides, experiments in wind tunnels are often performed due to their outstanding benefits. Wind tunnel tests allow obtaining data about the performance of parachutes with controlled environmental conditions and supporting equipment which is easier to operate and monitor than flight tests [15]. Therefore, this method is an effective application to compare different types of parachutes by measuring the coefficient of force (lift, drag, tangential, ...), angle of oscillation and so on [6].

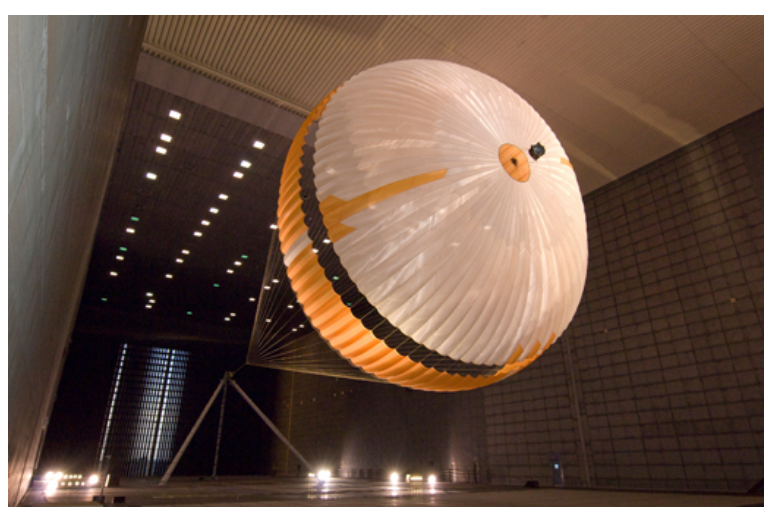

Fig. 3. A parachute in flight-qualification testing in the wind tunnel at NASA Ames Research Center, Calif. (2009) Source: NASA/JPL-Caltech.

Depending on primary purposes of tests, a kind of wind tunnel is selected to experience parachute models. The wind tunnel which is suited for collecting qualitative aerodynamic data with high accuracy is closed-throat (or closed test section), full-return due to a uniform velocity distribution in the test section. However, in the present research, due to lack of facilities, the open-return wind tunnel with a closed optically transparent test section is utilized. Therefore, generally, a drawback appearing in the experimental process is not convenient for changing the parachute configuration and models [6].

\section{Experimental Setup}

\subsection{Parachute Models}

The investigations were performed with two solid cloth canopy parachutes namely a flat circular and an extended skirt $10 \%$ (Fig. 4) based on the category of T. W. Knacke [6]. All of the models' canopies had the same surface area, $0.042 \mathrm{~m}^{2}$ so their nominal diameter was equal to each other. The number of gores and the suspension lines was 16. The lines were attached to a stationary mounting system including a RC benchmark loadcell Model 1520. The sensor allows measuring a force up to $5 \mathrm{~kg}$.

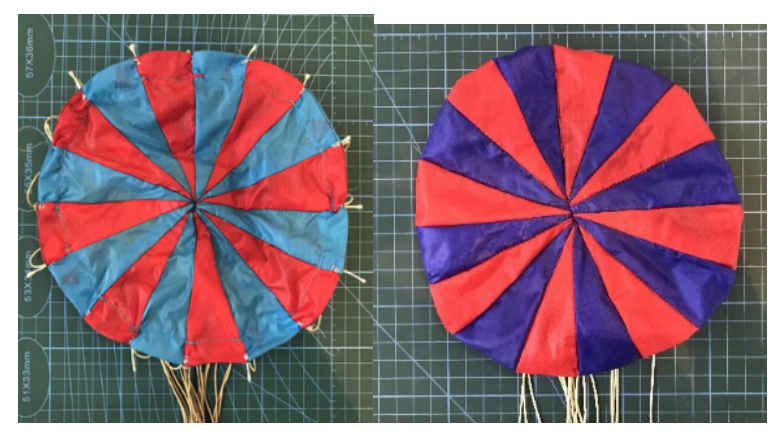

Fig. 4. Flat circular (left) and extended skirt 10\% (right) canopies (top view)

In the first series of experiments for evaluating the influences of the canopy shape on the drag coefficient, the canopies of parachute models were constructed by the same fabric, MIL-C-7030 Type I known as ripstop nylon $1.1 \mathrm{oz}$. The suspension lines were constructed by nylon strings with the diameter of $1 \mathrm{~mm}$ and the length of $0.23 \mathrm{~m}$, which resulted in a ratio between the length of suspension lines and the nominal diameter equal to $1\left(L_{e} / D_{o}\right)$.

The aim of the second series is to study the relationship between the length of suspension lines and the drag coefficient. Each model of two canopy types in this series maintained most specifications of the canopy in the previous series, there was no change in materials applied for the components of the model. Except for the suspension line length, the ratio between it and the nominal diameter $L_{e} / D_{o}$ was changed in the range from 0.6 to 1.5 , the drag force of each model was measured with a wind speed of $8 \mathrm{~m} / \mathrm{s}$.

\subsection{Experimental Setup}

In the present study, the parachutes were tested in a low-speed wind tunnel having similar specifications as the wind tunnel used in the study of Jin et al. at Tongji University $[10,12]$ (Table 1).

Table 1. The specification of wind tunnel

\begin{tabular}{ll}
\hline Dimensions $(\mathrm{m})$ & $1.0 \times 0.4 \times 0.5$ \\
\hline Maximum wind speed & $\approx 0.1 \mathrm{M}(30 \mathrm{~m} / \mathrm{s})$ \\
\hline
\end{tabular}

The experimental setup of parachute testing is presented in Fig. 5 while loadcell was connected to a data-acquisition circuit and then a personal computer installed data-acquisition software. Before starting experiments, wind tunnel and loadcell need to be calibrated. 


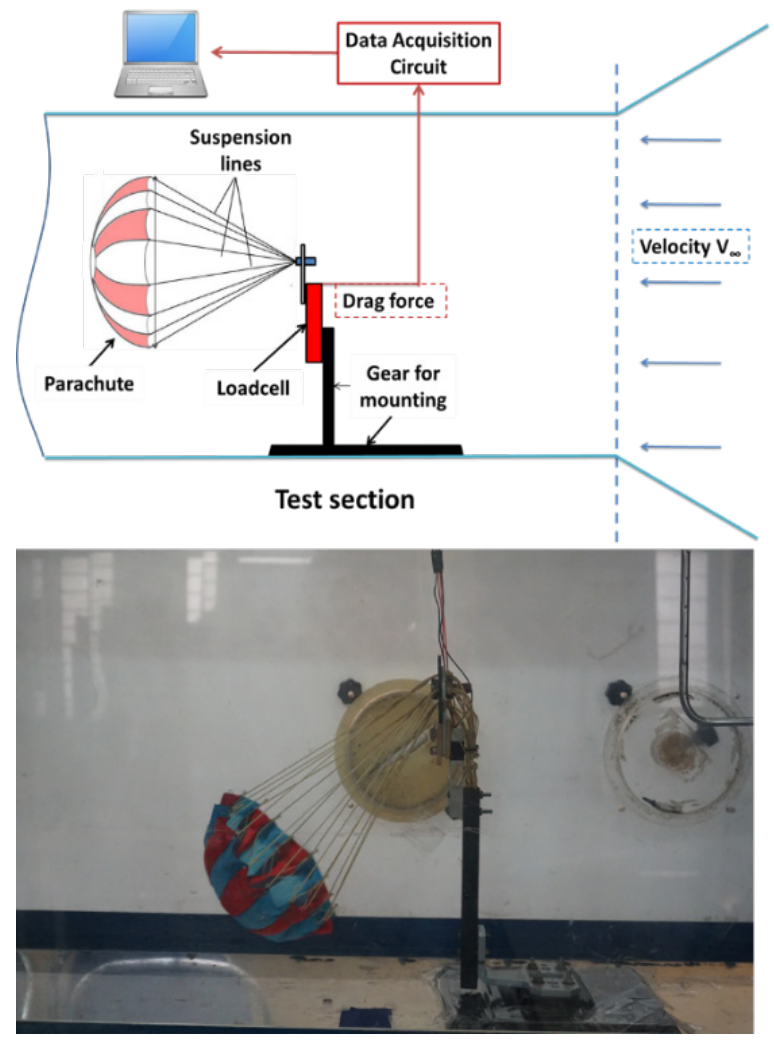

Fig. 5. Experiment setup of parachute testing in the wind tunnel.

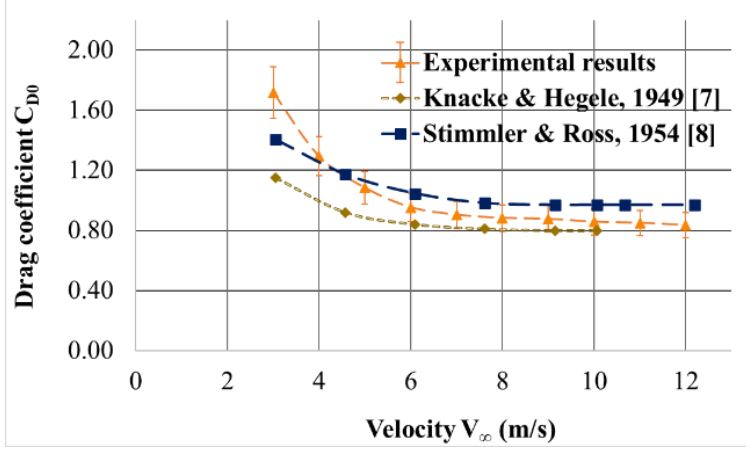

Fig. 6. The drag coefficient vs. velocity plot of the flat circular parachute

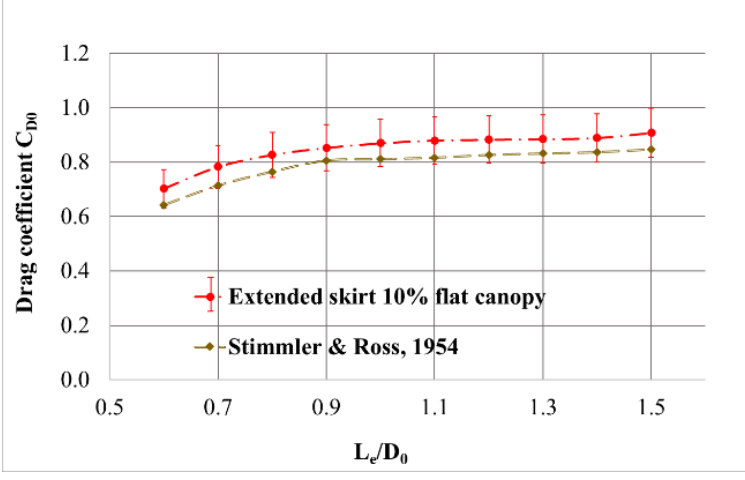

Fig 7. The drag coefficient vs. the rate of $L_{e} / D_{o}$ plot of the flat circular parachute.
In the first part, each model of two parachute types, flat circular canopy and extended skirt 10\% flat canopy, was tested with the freestream velocity changing in a range of the $3 \mathrm{~m} / \mathrm{s}$ to $12 \mathrm{~m} / \mathrm{s}$. At a certain speed, about 5000 samples of drag force which the loadcell transfers to the computer as the pulses of the signal were taken to calculate time-average drag force and finally drag coefficient was derived from the experimental force by equation (2).

In the second part, all models with the ratio $L_{e} / D_{o}$ between a 0.6 and 1.5 are put into the uniform flow field with a speed of $8 \mathrm{~m} / \mathrm{s}$. The method to collect data about the drag force and determine the coefficient is similar to the first part. The average value of drag coefficients for each ratio between the length of the suspension line and the nominal diameter was compared to the figure when $L_{e} / D_{o}$ was equal to 1.0.

\subsection{Validation}

The flat circular parachute is as the model which validated the accuracy and compatibility of the experimental setup for determining the drag force of parachutes in horizontal wind tunnels.

In the case of determining only the drag coefficient of the flat circular parachute with the rate of $L_{e} / D_{o}=1$ (Fig. 6), experimental results generally show a decreasing tendency when the freestream velocity increases from $3 \mathrm{~m} / \mathrm{s}$ to $12 \mathrm{~m} / \mathrm{s}$. The coefficients of drag force corresponding to the wind speed over $4 \mathrm{~m} / \mathrm{s}$ are approximate to the results presented in $[7,8]$ with percent errors less than $10 \%$. For the values of drag coefficient in the experiments to consider the influence of the suspension-line length, Figure 7 shows that the change of the drag coefficient measured in the present study is the same as the results published in [8]. errors remain under $10 \%$.

Therefore, the method and setup of this research can be suited for studying further aerodynamic characteristics of parachutes.

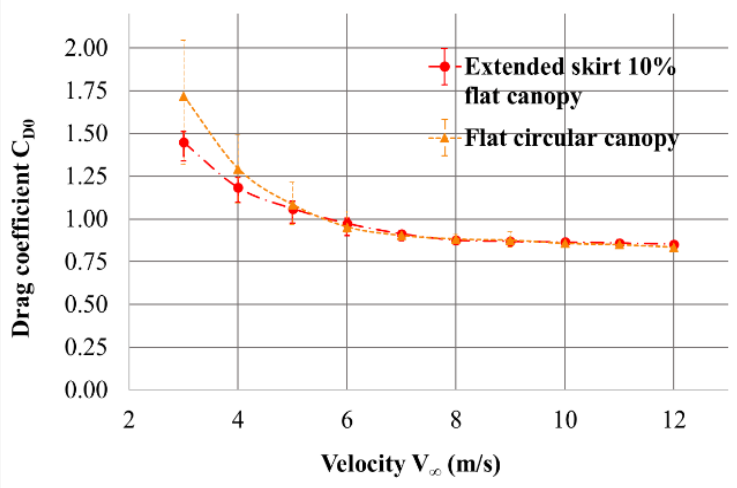

Fig. 8. The drag coefficient vs. velocity plot of two types of canopies. 


\section{Results and Discussion}

\subsection{Influence of Canopies}

The condition of the atmosphere maintained at the temperature of $25 \pm 1^{\circ} \mathrm{C}$ and pressure of $1.01 \times 10^{5} \mathrm{~Pa}$. Figure 8 displays the comparison of the curve of the drag coefficient versus the freestream velocity between two types of parachute canopies. There is a decreasing trend in the drag coefficient if the velocity increases, the drag coefficient gradually went down to about 0.85 at the inlet stream over $10 \mathrm{~m} / \mathrm{s}$.
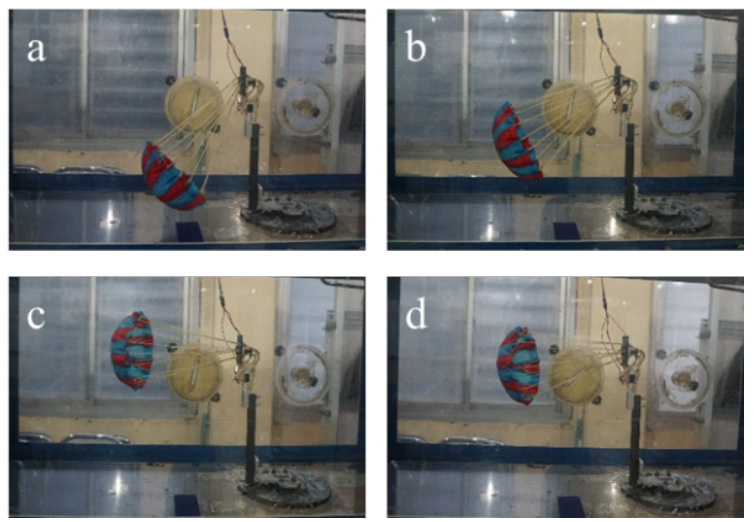

Fig. 9. Flat circular canopy at various freestream velocities, a) $3 \mathrm{~m} / \mathrm{s}$, b) $6 \mathrm{~m} / \mathrm{s}$, c) $9 \mathrm{~m} / \mathrm{s}$, d) $12 \mathrm{~m} / \mathrm{s}$.
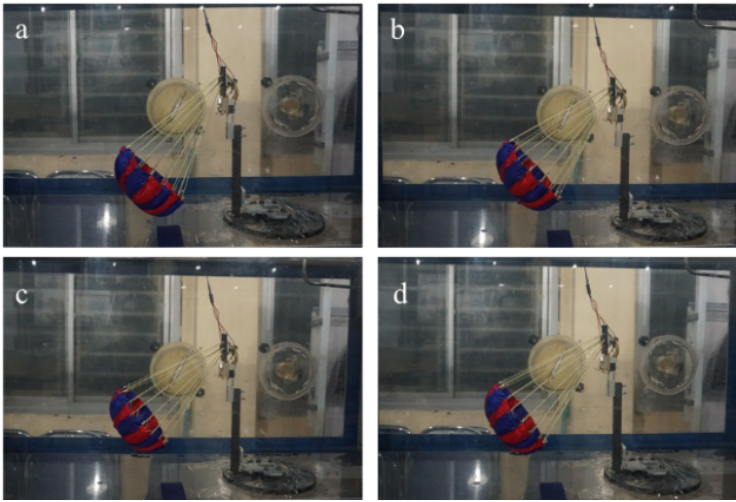

Fig. 10. Extended skirt $10 \%$ flat canopy at various freestream velocities, a) $3 \mathrm{~m} / \mathrm{s}$, b) $6 \mathrm{~m} / \mathrm{s}$, c) $9 \mathrm{~m} / \mathrm{s}$, d) $12 \mathrm{~m} / \mathrm{s}$.

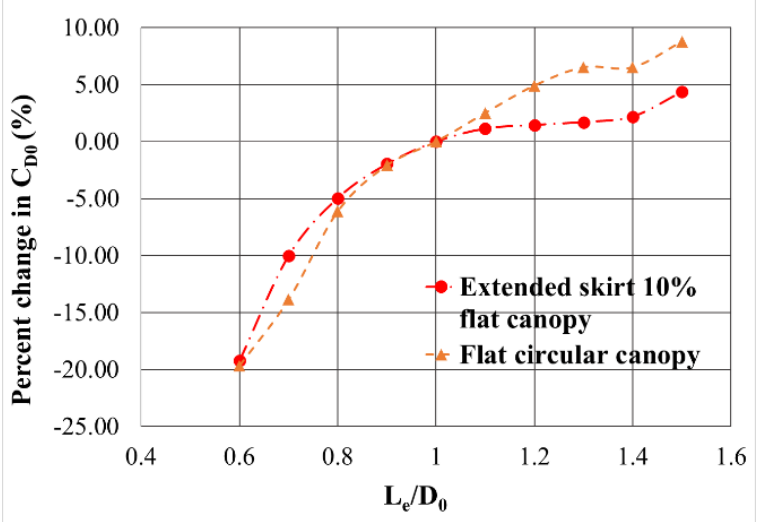

Fig. 11. The percent change in drag coefficients of two types of parachute canopies
At the freestream velocity lower than $5 \mathrm{~m} / \mathrm{s}$, there is a considerable difference in the drag coefficient of the two canopy types. To be more specific, the figure of the flat circular canopy, which is 1.72 , is more 0.27 than of the extended skirt $10 \%$ flat canopy. However, at the high velocity, the extended skirt $10 \%$ flat canopy has higher time-averaged drag forces than the flat circular canopy although the different level in drag coefficients of two parachute types is pretty small around 0.1 .

At the low speed, the reason for the canopies' instability is that pressure on the inner canopies' surface is not uniform and high enough to maintain the stable structure of canopies and the high tension of the suspension lines. Hence, the skirt of canopies can be expanded, that is the projected area $S_{p}$ is larger than the nominal area $S_{o}$ and a little drag force is added to the total one. In contrast, high pressure putting on the inner surface at high speeds makes the canopies more stable, the projected area $S_{p}$ is approximate to the nominal area $S_{o}$. Therefore, the drag coefficient at the low inlet velocity is greater than at the high values with the constant nominal area.

The difference in the drag coefficient of two canopy types is derived from the stability of the canopy structure when the turbulence occurs. If we considered the parachutes and the flow field in the test section along the horizontal axis, there is the turbulent flow occurring behind the canopies. In terms of the flat canopy, the maximum projected diameter is the diameter at the edge of the canopy skirt. The turbulent flow affects directly that edge, which makes the canopy expand. Meanwhile, the flat canopy's edge with the extended skirt $10 \%$ is shrunk, the diameter at the edge is smaller than the maximum inflated diameter of the parachute. Thus, this protects the parachute's structure from the negative effect of the turbulence, the amount of inner pressure is controlled and not changed significantly.

In addition, when the wind speed was larger than $8 \mathrm{~m} / \mathrm{s}$, the oscillation of parachutes appeared and became more intensive. Two parachute models also oscillated with small angles at a speed less than $6 \mathrm{~m} / \mathrm{s}$.

\subsection{Effect of the Length of Suspension Line}

The experimental process was performed in the air at $22 \pm 2{ }^{\circ} \mathrm{C}$ and $1.01 \times 10^{5} \mathrm{~Pa}$. Since the freestream velocity is kept at the constant medium value of $8 \mathrm{~m} / \mathrm{s}$, the oscillation of parachutes is not too large. Figure 11 illustrates the coefficient of drag force grows if the length of the suspension lines increases, which means there is an increase in the ratio between that length and the nominal diameter. For the range of the ratio $L_{e} / D_{o}$ between 0.6 and 1.0, the drag coefficient of two types goes up significantly, the additional proportion of the flat circular canopy is less than of the extended skirt 
$10 \%$ flat canopy.

Nevertheless, it is witnessed that there is a remarkable difference in the plot of the percent change of the drag coefficient between two models when $L_{e} / D_{o}$ is from 1.0 to 1.5 . While the figure of extended skirt $10 \%$ flat models only increases slightly and reached nearly $4.4 \%$, the flat circular canopy experienced a dramatic growth in the drag coefficient. At $L_{e} / D_{o}=1.5$, the increasing percentage of the flat circular parachute in comparison with the ratio $1.0 \mathrm{was}$ about twice as much as the proportion of the extended skirt one.

In previous studies, Knacke proved that the drag coefficient of the flat circular parachute was possible to increase continuously for $L_{e} / D_{o}$ up to 2.0 [6]. Meanwhile, the extended skirt $10 \%$ flat has little growth in its drag coefficient, approximate to $2 \%$ at the ratio $L_{e} / D_{o}$ greater than 1.3 [6], [16]. It is difficult to determine exactly small developments in drag force due to the limit of the accuracy in the measurement by loadcell, in addition, the flexibility of canopy and instability of flow field through it have a significant impact on the drag force. Therefore, there are some abnormal points in the curve such as the figure at $L_{e} / D_{o}=1.4$ on the plot of the flat circular canopy and at 1.5 on the curve of the extended skirt $10 \%$ parachute.

The change in the line length causes the expansion or the shrinkage of the parachute canopies. As mentioned above, the specific kind of parachute has the optimizing length for its suspension lines, whose ratio $L_{e} / D_{o}$ to the nominal area is about 1.0. Declining the suspension lines' length leads to shrinking the area of canopies, as a result, the drag force reduces certainly. In addition, the forebody wake created by the mounting stationary also contributes to decreasing the total drag force.

The primary reason to explain the difference between two curves of the parachute in Fig. 11 is similar to the previous cases (4.1. Influence of canopy shapes). If the suspension lines are extended, consequently the canopies will inflate wider and simultaneously there is a dramatic growth in the drag force. The inflation of the flat circular canopy totally follows the phenomenon. However, the structure of the extended skirt $10 \%$ flat canopy only allows an expansion to a certain dimension. To be more specific, the lower part or the skirt whose diameter is equal to $0.1 D_{o}$ restricts the opening of the canopy. Therefore, the projected area of that canopy is nearly fixed, in other words, the amount of the produced drag force remains stable or increases slightly.

\section{Conclusion}

In conclusion, in this paper, the effects of canopy shape and the suspension-line length on the drag coefficient of parachutes were investigated. The results show that the flat circular canopy produced the drag force little less than the extended skirt $10 \%$ models when the freestream velocity is over $5 \mathrm{~m} / \mathrm{s}$. Based on the observation during the experimental process, it is concluded that the parachutes applying in recovery systems in UAVs should be designed to have the rate of descent from $5-7 \mathrm{~m} / \mathrm{s}$. That rate guarantees the safety requirement for UAVs and the performance of parachutes, that is the small weight, high drag and stability with small oscillation.

With $0.6<L_{e} / D_{o}<1.0$, two types of parachutes have the increasing trend in percent change of drag although the figure of the flat circular canopy is smaller than the extended skirt $10 \%$ parachute. However, in this case $1.0<L_{e} / D_{o}<1.5$, the additional percentage of drag coefficients of the flat circular model is more than the extended skirt $10 \%$ flat parachute.

The paper only investigated some factors which have the impact on the drag coefficient of parachutes. It is necessary to investigate other factors to consider completely the characteristics and performance of parachutes before applying them for recovery systems in UAVs. Furthermore, future studies can take into consideration the porosity of material and stability in the descent motion, the parachute models are recommended such as cross, ribbon or disk-gap band parachutes.

\section{References}

[1]. Unmanned Aircraft Systems (UAS), International Civil Aviation Organization, Montréal, Canada, 2011, pp. 3.

[2]. Marcus Eriksson, Patrick Ringman, Launch and recovery systems for unmanned vehicles onboard ships, Centre for Naval Architecture, 2013.

[3]. Operation of Small Unmanned Aircraft Systems Over People, Federal Aviation Administration, Department Of Transportation, USA, 2019.

[4]. T. Wyllie, Parachute recovery for UAV systems, Aircraft Engineering and Aerospace Technology, vol. 73, pp. 542-551, 2001.

https://doi.org/10.1108/00022660110696696

[5]. Vasile PRISACARIU, Sebastian POP, Ionică CÎRCIU, Recovery system of the multi-helicopter uav, in Review of the Air Force Academy, Braşov, Romanian, Henri Coanda Air Force Academy Publishing House, 2016, pp. 91-98. https://doi.org/10.19062/1842-9238.2016.14.1.13

[6]. T. W. Knacke, Parachute Recovery Systems, China Lake, CA, US, 1991.

[7]. T. W Knacke and A. M. Hegele, Comparison Tests of Various Types, U.S. Air Force,USA, Tech.Rep. Model 
Parachutes, 1949.

[8]. J. F. Stimmler and R. J. Ross, Drop Tests of 16,000 sq. in. Model Parachutes, U.S. Air Force, USA, Tech.Rep. Model Parachutes, Volume VIII, 1954.

[9]. H. Johari, A. Levshin, Interaction of a line vortex with a round parachute canopy, Journal of Fluids and Structures, p. 1258-1271, 2009. https://doi.org/10.1016/j.jfluidstructs.2009.07.005

[10]. Zhe-Yan Jin, Sylvio Pasqualini, Bo Qin, Experimental investigation of the effect of Reynolds number on flow structures in the wake of a circular parachute canopy, Acta Mechanica Sinica, 2014.

http://doi.org/10.1007/s10409-014-0058-1

[11]. Yu Li, Cheng Han, Zhan Ya'nan, Li Shaoteng, Study of parachute inflation process using fluid-structure, Chinese Journal of Aeronautics, vol. 27, pp. 272-279, 2014.

https://doi.org/10.1016/j.cja.2014.02.021

[12]. Zheyan Jina, Sylvio Pasqualini, Zhigang Yang, Experimental investigation of the flow structures in the wake of a, European Journal of Mechanics B/Fluids, pp. 70-81, 2016.

https://doi.org/10.1016/j.euromechflu.2016.07.008

[13]. Keith R. Stein, Richard J. Benney, Fluid-Structure Interactions of a Round Parachute: Modeling and
Simulation Techniques, Journal of Aircraft, p. 802, 2001.

https://doi.org/10.2514/2.2864

[14]. Xue YANG, Li YU, Min LIU, Haofei PANG, Fluid structure interaction simulation of supersonic parachute inflation by an interface tracking method, Chinese Journal of Aeronautics, vol. 33, pp. 16921702, 2020.

https://doi.org/10.1016/j.cja.2020.03.005

[15]. DJ.Cockrcll, The Aerodynamics of Parachutes, List of National Distribution Centres, NATO Science and Technology Organization., 1987.

[16]. T. W. Knacke, L. L. Dimmick. , Design Analysis of Final Recovery Parachutes for B- 70 Encapsulated Seat and the USD-5 Drone, Ohio : USAF, 1962.

[17]. Ashim Panta, Simon Watkins, Reece Clothier, Dynamics of a small unmanned aircraft parachute system, Journal of Aerospace Technology and Management, vol. 10, 2018. https://doi.org/10.5028/jatm.v10.752

[18]. Sylvio Pasqualini, Zheyan Jin, Zhigang Yang, Measurement of the flow structures in the wakes of different types of parachute canopies, Acta Mechanica Sinica, 2017.

https://doi.org/10.1007/s10409-017-0710-7 